\title{
Kelvin-Helmholtz Instability: Lessons Learned and Ways Forward
}

\author{
A. $\operatorname{Masson}^{1}\left(\mathbb{D} \cdot\right.$ K. Nykyri $^{2}$
}

Received: 26 October 2017 / Accepted: 16 April 2018 / Published online: 24 April 2018

(C) The Author(s) 2018

\begin{abstract}
The Kelvin-Helmholtz instability (KHI) is a ubiquitous phenomenon across the Universe, observed from $500 \mathrm{~m}$ deep in the oceans on Earth to the Orion molecular cloud. Over the past two decades, several space missions have enabled a leap forward in our understanding of this phenomenon at the Earth's magnetopause. Key results obtained by these missions are first presented, with a special emphasis on Cluster and THEMIS. In particular, as an ideal instability, the KHI was not expected to produce mass transport. Simulations, later confirmed by spacecraft observations, indicate that plasma transport in KelvinHelmholtz $(\mathrm{KH})$ vortices can arise during non-linear stage of its development via secondary process. In addition to plasma transport, spacecraft observations have revealed that KHI can also lead to significant ion heating due to enhanced ion-scale wave activity driven by the KHI. Finally, we describe what are the upcoming observational opportunities in 20182020, thanks to a unique constellation of multi-spacecraft missions including: MMS, Cluster, THEMIS, Van Allen Probes and Swarm.
\end{abstract}

Keywords Kelvin Helmholtz instability · Earth's magnetopause · Cold dense plasma sheet $\cdot$ Cluster $\cdot$ THEMIS $\cdot$ MMS

\section{Introduction}

A fundamental question in space physics is the penetration of solar wind plasma, momentum and energy into the Earth's magnetosphere, depending on solar activity. Fundamental works by Dungey $(1961,1963)$ and data collected by early space missions have led to the following textbook vision. Southward oriented interplanetary magnetic field (IMF) favours the occurrence of magnetic reconnection at the subsolar magnetopause ultimately leading to storage of magnetic energy and reconnection in the magnetotail. During Northward oriented IMF, magnetic reconnection at this location is much less favoured, occurring mostly

\footnotetext{
A. Masson

Arnaud.Masson@esa.int

1 European Space Agency, ESAC, SCI-OPD, Madrid, Spain

2 Centre for Space and Atmospheric Research, Embry-Riddle Aeronautical University, Florida, USA
} 
tailward of the cusps, while solar wind plasma penetration is observed at the Low Latitude Boundary Layer or LLBL (e.g., Eastman et al. 1976) and in the central plasma sheet (e.g., Baumjohann et al. 1989). Hence, other physical phenomena have been proposed including high-latitude reconnection (e.g., Song and Russell 1992), and the Kelvin-Helmholtz Instability (KHI) (Axford and Hines 1961). These two phenomena can operate at the same time (e.g., Taylor et al. 2008).

In Sects. 3 and 4, we will present recent results on the KHI that challenge certain aspects of this textbook vision. The vast majority of these results are based on data collected by the multi-spacecraft ESA Cluster mission (Escoubet et al. 2001) and the NASA THEMIS mission (Angelopoulos 2008). A quick reminder on the KHI phenomenon itself and the open questions prior to the launch of these missions are presented in the next section. Section 5 presents the first observational evidence of cross-scale energy transport within a $\mathrm{KH}$ vortex, and its implications. Unique observational opportunities in 2018-2020 timeframe of this phenomenon with MMS, Cluster, THEMIS, Van Allen Probes and Swarm are finally presented in Sect. 6.

\section{KHI Before the Cluster and THEMIS Era}

The Kelvin-Helmholtz instability can develop at the interface of two fluids in the presence of a velocity shear. This phenomenon was first described more than a century ago by Helmholtz (1868) and Kelvin (1871). For non-viscous fluids, there is no instability threshold and a velocity shear is always unstable (e.g., Chandrasekhar 1961). However, in a magnetized plasma, the plasma compressibility and the magnetic tension force can have a stabilizing effect (Miura and Pritchett 1982). Hence, a minimum value of the velocity shear is required for the instability to occur, which depends on the initial conditions of the flow. An analytical expression of this instability threshold can be obtained for an ideal MHD plasma in an incompressible media (e.g. Smets et al. 2002). It can also be demonstrated that, in the incompressible media approximation, the component of the instability wave vector perpendicular to the interface is purely imaginary. Hence, the perturbation is a surface wave at the interface. In a more realistic approach, the KH eigen modes decay away from the shear boundary, confining it as a surface wave on the interface. Non-linear evolution of the KHI can result in vortices.

In fluids, KHI vortices are observed in many environments including: oceans (e.g., Smyth and Moum 2012), at the edge of clouds (e.g., Houze 2014), or in the atmosphere of giant planets (e.g. Saturn, Jupiter). In collisionless space plasmas, KHI occurs in various regions of the solar system, for instance: at the magnetopause of various planets like Mercury, possibly Venus, Jupiter and Saturn (e.g., Johnson et al. 2014), the edge of coronal mass ejections (Foullon et al. 2011; Nykyri and Foullon 2013) and more generally in the solar atmosphere and the solar wind (e.g., Mishin and Tomozov 2016). KHI is also considered to play an important role in various astrophysical objects such as the Orion nebula (Berné et al. 2010), the pulsar wind (Bucciantini et al. 2005) or around quasar (e.g., Lobanov and Zensus 2001).

At Earth, KHI is observed at the magnetopause where a velocity shear exists due to the magnetosheath plasma flowing along the magnetopause. This plasma is indeed faster but also denser and colder than the magnetospheric plasma (e.g., Belmont and Chanteur 1989). The Earth's magnetopause offers a unique testbed where all scales of this phenomenon can nowadays be investigated: fluid, ion and electron scales. Furthermore, ground-based instrumentation and various magnetospheric missions enable to capture the impact of this phenomenon in terms of plasma entry in the plasma sheet, the generation of ULF waves and its consequences in the ionosphere such as field aligned currents. 
Before the launch of the Cluster mission in 2000 and THEMIS in 2007, many questions remained open about this phenomenon including:

- Does KHI lead to the development of rolled-up vortices along the magnetopause?

- Are they rare or a common feature?

- Is there a dawn-dusk asymmetry? if yes what causes it?

- Does the Kelvin-Helmholtz instability only occurs during Northward IMF and near the LLBL?

- What are the physical mechanisms within a KHI vortex allowing magnetosheath plasma to enter or magnetospheric plasma to exit the magnetosphere?

- How does this phenomenon develop from birth to collapse along the magnetopause?

- How is magnetic reconnection initiated within KH rolled-up vortices?

- What is the contribution of the KHI vs. magnetic reconnection in terms of plasma entry?

As we will see in the following sections, most of these questions have been answered or tackled thanks to Cluster and THEMIS data, complemented by other missions like Geotail and Double Star.

\section{Multi-spacecraft Vision}

During a prolonged period of northward IMF, the Cluster spacecraft revealed that KH waves can roll-up and evolve into 40,000-55,000 km wide vortices along the Earth's magnetopause (Hasegawa et al. 2004), see left panel of Fig. 1. This observation is key as according to MHD simulations, plasma transport across the magnetopause can occur within or at the edge of such fully developed vortices. Different scenarios of plasma transport have been proposed over the years, including: magnetic reconnection (e.g. Nykyri and Otto 2001; Nykyri et al. 2006), diffusive particle transport via turbulence (e.g. Matsumoto and Hoshino 2004; Nakamura et al. 2004; Cowee et al. 2009, 2010; Stawarz et al. 2016) and anomalous transport due the conversion of KH surface waves to kinetic Alfvén waves (Chaston et al. 2007); see also Sect. 5.

But before discriminating between these different candidates, more data had to be analysed to know if these rolled-up vortices were a common feature during Northward IMF or not. Cluster, with its nominal polar orbit, is not optimal to capture a large number of events at the LLBL. However, a 3-D MHD simulation by Takagi et al. (2006) suggested that rolled-up vortices could be detected by a single spacecraft crossing the magnetopause. This simulation indeed demonstrates that at a certain radial distance from the centre of a rolled-up vortex, tenuous plasma must rotate faster than a denser plasma for the force balance in the radial direction to be maintained. This result confirmed previous predictions based on 2-D MHD simulations (Nakamura et al. 2004). Hence, the tailward speed of at least a fraction of the low-density magnetospheric plasma must exceed the speed of the dense magnetosheath plasma. As this feature is characteristic of a fully developed $\mathrm{K}-\mathrm{H}$ vortex and potentially detectable by a single spacecraft, Takagi et al. (2006) suggested to use it as a tracer of the presence of such vortices.

Hasegawa et al. (2006) not only confirmed the presence of this signature in the Cluster data analysed in Hasegawa et al. (2004) but also presented a survey of the Geotail data collected from 1995 to 2003. This survey reveals that these vortices are not rare under northward IMF conditions. For each vortex detected, magnetosheath ions are systematically present on the magnetospheric side of the boundary. Hence, the KHI may play a significant role in the formation of the LLBL flank under Northward IMF. More precisely, this study 

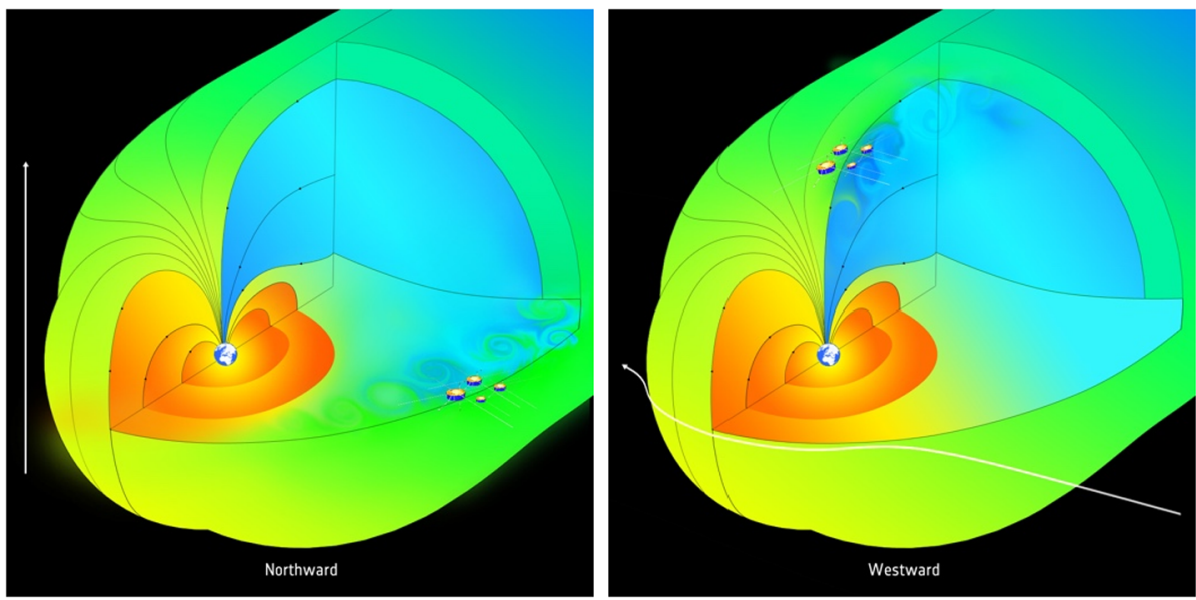

Fig. 1 Left panel: sketch of the magnetosphere with Kelvin-Helmholtz vortices generated on the dusk flank during a prolonged period of northward IMF. Right panel: sketch of Kelvin Helmholtz vortices developing at high-latitudes near the northern cusp during strongly westward IMF. Please note that this is an artistic illustration of the Kelvin-Helmholtz waves observed at the high-latitude magnetopause by Hwang et al. (2012). These waves were observed at the interface between the magnetosheath and high-latitude closed field line region and not at the interface between the sheath and the lobe open field lines as this sketch may suggest. (credit: ESA)

lists 19 events when rolled-up vortices were found under northward IMF with a vast majority during a prolonged period of strong Northward IMF, while no dawn-dusk asymmetry was found. According to Hasegawa et al. (2006), this number shall be considered as a lower bound of the magnetopause rolled-up vortices occurring during that time frame, for two main reasons. First, the high-speed/low-density signature mentioned above is not always present during a rolled-up vortex (Takagi et al. 2006). Second, the time resolution of the plasma instruments on Geotail is only $12 \mathrm{~s}$.

Based on the same methodology, similar surveys have been conducted with data collected by the Double Star TC-1 spacecraft (Taylor et al. 2012) and the THEMIS probes B and C (Lin et al. 2014). ${ }^{1}$ These surveys confirmed the conclusions of Hasegawa et al. (2006): KHI rolled-up vortices are not rare under northward IMF conditions and may contribute significantly to the entry of magnetosheath plasma in the magnetosphere. However, based on 34 events collected by TC- 1 and Geotail, Taylor et al. (2012) noted a possible dawn-dusk asymmetry, with a preferential growth on the dusk side in $62 \%$ of the cases analysed $(70 \%$ for TC1 events only). No particular dawn-dusk asymmetry is reported by Lin et al. (2014). It has to be noted that a revisit of THEMIS case studies events led to the following conclusion: the lower density and faster than sheath marker for KHI rolled up vortices proposed by Takagi et al. (2006) shall be used with great caution for plasma observations at the dayside magnetopause, especially sunward of the terminator (Plaschke et al. 2014). In other words, a few of the events listed in the above-mentioned studies may not be genuine crossing of KHI rolled up vortices.

Furthermore, none of these studies took into account if the horizontal component of the IMF was in a Parker spiral (PS) or ortho-Parker spiral (OPS) orientation. MHD simulations

\footnotetext{
${ }^{1}$ Lin et al. (2014) made use of the events reported by Hasegawa et al. (2006), Taylor et al. (2012) plus extra Geotail and Cluster events reported by Hasegawa et al. (2004), Nishino et al. (2011) and Moore (2012).
} 
under PS and OPS orientations and under various solar wind plasma conditions suggest that KHI favours the dawn-flank during PS orientation when source region is the dawndusk terminator. For the PS orientation, the magnetic field tension is indeed smaller at dawn when compared to dusk sector (Nykyri 2013). One of the conclusions of this study is that statistically, the KHI formation may favour the dawnside flank, due to the fact that the IMF is mostly in the Parker spiral orientation.

A revisit of a statistical survey of 7 years of THEMIS data (Kavosi and Raeder 2015), taking this aspect into account, tends to confirm this prediction observationally (Henry et al. 2017). The normalized occurrence rates showed clear dawn flank preference during PS IMF, and dusk flank preference during northward IMF (Henry et al. 2017). This study also found that the KHI was observed at dusk-sector for higher solar wind speeds when compared to the dawn, which was attributed to the increased magnetic tension at the dusk flank. To be able to overcome increased magnetic tension, the higher velocities are indeed required. This dawn-dusk asymmetry of the KHI formation towards dawn may also be linked to the plasma sheet asymmetry of cold-component ions, which are heated more on the dawnside plasma sheet (Wing et al. 2005). Upcoming conjunctions of MMS, Cluster and THEMIS may help providing ground truth of this important aspect as it will allow simultaneous monitoring of both flanks during the same solar and IMF conditions (see Sect. 6.2).

\section{KHI Under Different IMF Conditions}

Crossing of a KHI by Cluster at the magnetopause is detailed by Nykyri et al. (2006). Two remarkable features are presented in this case study. For the first time, signatures of magnetic reconnection within $\mathrm{KH}$ vortices are observed. The second noticeable feature is the IMF $\mathrm{B}_{z}$ : it is only slightly northward and the IMF is mostly in Parker-spiral orientation. In other words, plasma transport by KHI from the magnetosheath to the magnetosphere may happen not only during strongly northward IMF conditions. Any configuration that satisfies the $\mathrm{KH}$ onset condition could lead to the instability. For the ideal MHD case in an incompressible media, the instability criterion is (e.g., Smets et al. 2002)

$$
\left(\mathbf{k}_{T} \cdot \Delta \mathbf{v}\right)^{2}>\left(1 / \rho_{M S H}+1 / \rho_{M S P}\right)\left(\rho_{M S H}\left(\mathbf{k}_{T} \cdot \mathbf{v}_{A M S H}\right)^{2}+\left(\rho_{M S P}\left(\mathbf{k}_{T} \cdot \mathbf{v}_{A M S P}\right)^{2}\right)\right.
$$

where $\Delta \mathbf{v}$ is the shear velocity. MSH and MSP stand for magnetosheath and magnetosphere respectively; $\mathbf{k}_{T}$ is the wave vector component tangential to the interface, $\mathbf{v}_{A M S H}$ is the Alfvén velocity in the magnetosheath and $\mathbf{v}_{A M S P}$ in the magnetosphere.

In a more realistic case, the $\mathrm{KH} k$-vector will assume a direction to maximize the onset criteria. For example, the best comparison with data and simulation in Nykyri, 2006-event was obtained when $k$-vector was tilted by 35 degrees from the initial shear flow plane. This result was later confirmed with 3-D simulations of the same event by Adamson et al. (2016). Furthermore, the onset criteria is derived assuming infinitely thin boundary, and the real magnetopause has a finite thickness. Therefore, there is a typical minimum wavelength for KH wave growth (Miura and Pritchett 1982).

Further analysis of the Cluster and THEMIS magnetopause crossings near the flanks of the LLBL, led to the detection of KH vortices even during southward IMF conditions (Hwang et al. 2011; Yan et al. 2014), confirming previous observations (Mozer et al. 1994; Kawano et al. 1994) and 3-D MHD simulations (e.g., Claudepierre et al. 2008). In the case study of Hwang et al. (2011), Kelvin-Helmholtz waves were found to be much less stable, due to the fluctuations induced by magnetic reconnection in the sub-solar region and flux ropes drifting along the magnetopause. 
The same authors then investigated the possible presence of KHI during strongly dawnward IMF conditions (Hwang et al. 2012). For the first time, they reported the in-situ observations of Kelvin-Helmholtz waves along the magnetopause at high latitudes, near the northern duskward cusp (see right panel of Fig. 1). Recently, Ma et al. (2016) also found KH waves at high-latitudes using Cluster data. These observations confirm predictions made by past MHD simulations (e.g. Farrugia et al. 1998).

Another IMF magnetic configuration was investigated with multiple THEMIS spacecraft while the IMF was moving from radial to southward pointing (Grygorov et al. 2016). A well-developed rolled up $\mathrm{KH}$ vortex was observed very close to the subsolar point $\left(Y_{G S M} \sim 4.5 \mathrm{R}_{E}\right)$, at the inner edge of the LLBL. It was attributed to the intermittent nature of reconnection which leaves the time for a creation of the LLBL and the development of $\mathrm{KH}$ vortices at its inner edge.

All these results change our apprehension of this phenomenon. Indeed, early studies of the KHI had been investigated almost exclusively near the LLBL, i.e. equatorial flanks of the magnetopause, and during northward IMF. The motivations were twofold: investigate its possible role in the formation and the thickening of the LLBL (Mitchell et al. 1987) and the formation of the Cold Dense Plasma Sheet or CDPS (e.g. Fujimoto et al. 1997), during northward IMF.

If one only considers the IMF orientation in the KHI onset condition, the purely northward and southward IMF should give the same $\mathrm{KH}$ growth rate. However, the main difference for the northward IMF vs. southward IMF driven KH dynamics comes from 3-D aspects: a system which is unstable to both $\mathrm{KH}$ modes and tearing modes (linear stage of magnetic reconnection) in the same scale requires that velocity shear and magnetic shear are not aligned, otherwise either the KH mode or the tearing mode would be stabilized (Chen et al. 1997). This situation can happen for example during southward IMF at the magnetopause in presence of an adequate shear-flow in agreement with the KHI onset condition. The results of this interaction between the KHI and the tearing mode depend strongly on the initial and boundary conditions, namely, conditions where (1) KH modes represent the initial or primary process (Ma et al. 2014a) or (2) magnetic reconnection is the primary process (Ma et al. 2014b). Both of these will modify the other mechanism, which can lead to less regular periodic signatures of the plasma and field properties, making it more difficult to identify the KHI. On the other hand, the formation of the LLBL under northward IMF increases the density on the magnetospheric side and thus reduces the magnetospheric Alfvén speed (Sect. 4.3 in Hasegawa 2012). The presence of the LLBL may reduce the velocity jump across the magnetopause (by a factor of $\sim 2$ ), but since it can increase the magnetospheric density by an order of magnitude, resulting in reduced density asymmetry, the KHI onset condition can be satisfied more often for northward than for southward IMF conditions. This can be the other reason why $\mathrm{KH}$ waves are more often observed for northward IMF.

A survey of seven years of magnetopause crossings by the THEMIS spacecraft constellation confirm this aspect, statistically (Kavosi and Raeder 2015). During these seven years, these satellites crossed the magnetopause during around 960 hours, almost half of the time during northward IMF $(\sim 500 \mathrm{~h})$ and half of the time during southward IMF $(\sim 460 \mathrm{~h})$. Kelvin Helmholtz waves were found at the magnetopause around $35 \%$ of the time for northward IMF and (unexpectedly) $10 \%$ of the time during southward IMF. The overall occurrence rate is $19 \%$ for any solar wind and IMF conditions. This rate not surprisingly increases with solar wind speed (which increases the velocity shear), Alfvén Mach number and density. However, KHI was found to be mostly independent of IMF magnitude.

It is worth reminding that the orbit of these satellites lies roughly in the equatorial plane and cannot capture KHI developing at high latitudes such as the observations reported by 
Hwang et al., Ma et al. (2012, 2016) under strongly dawnward IMF. In other words, 19\% is most probably a lower limit of the presence of this phenomenon, regardless of the IMF orientation.

\section{First Observations of Cross-Scale Energy Transport Within a KH Vortex}

One of the pending problems in collisionless plasmas is to understand the plasma heating and transport across three fundamental scales: fluid, ion and electron. Moore et al. (2016) used Cluster observations with $80 \mathrm{~km}$ separation, and quantified the "cross-scale" energy transport from a fluid scale (wavelength of $36,000 \mathrm{~km}$ ) $\mathrm{KH}$ wave into an ion-scale fast magnetosonic wave packet (consisting of wavelengths of 200-2000 km). The existence of the fluid-scale Kelvin-Helmholtz instability was established by simulation-data comparison, and the ion-scale magnetosonic wave packet was identified by constructing the wave frequencies as a function of wave number in a plasma frame which was only possible because both $\mathrm{C} 1$ and $\mathrm{C} 4$ (which were only $\sim 80 \mathrm{~km}$ apart) observed the same wave packet with a time lag. The constructed experimental dispersion relation agreed with the dispersion of the theoretical MHD fast mode wave and at higher $k$-values agreed better with the kinetic magnetosonic mode solution obtained by the WHAMP (Rönmark 1982) dispersion solver assuming a 71 degrees' propagation angle between the wave $k$-vector and background magnetic field in the source region of the wave. The shell-like ion velocity distribution functions were determined to be the source of free energy for the kinetic magnetosonic waves, however the detailed mechanism generating these distribution functions is currently under investigation. It may have to do with particle motion in 3-D KH wave structure.

The energy budget of the cross-scale energy transport was calculated in the following way: the free kinetic energy per unit area, $K_{\text {in }}$ contained in the velocity shear between shocked solar wind and magnetospheric plasma was estimated using $K_{\text {in }}=1 / 2 \rho \Delta v_{M}^{2} \Delta x$, where $\rho$ is the average ion mass density and $\Delta v_{M}$ is the difference between the tailward velocities on the magnetosheath and magnetospheric side of the magnetopause. The $\Delta x=v_{\text {phase }} \Delta t$ where $\Delta t$ is the duration of the fast mode wave packet while $v_{\text {phase }}$ is the phase velocity of the $\mathrm{KH}$ wave determined by simulation-data comparison. The twisting of the magnetic field and compression of the magnetized plasma was calculated using $K_{\text {out }}=\left(\Delta B_{M}^{2} / 2 \mu 0+\Delta P_{\text {total }}\right) \Delta x$, where $\Delta B_{M}$ is the typical difference between the tailward and earthward magnetic field components created by the KHI. $\Delta P_{\text {total }}$ is the difference between the total pressures $\left(P_{\text {thermal }}+P_{\text {magnetic }}\right)$ on the magnetosheath and the magnetospheric side of the $\mathrm{KH}$ vortex. Please note that under steady solar wind conditions there should be total pressure balance at the magnetopause, but because $\mathrm{KHI}$ is a compressional instability it can produce total pressure variations. Hence, the energy budget estimates how much of the free energy, available in the velocity shear, is used by compression of the magnetic field and plasma as well as a look at the twisting of the tangential component of the magnetic field.

$K_{\text {in }}$ was estimated to be $840 \mathrm{GeV} / \mathrm{cm}^{2}$ and $K_{\text {out }}=820 \mathrm{GeV} / \mathrm{cm}^{2}$ leaving a surplus of about $20 \mathrm{GeV} / \mathrm{cm}^{2}$. The integrated Poynting flux during the magnetosonic wave packet was about 22 percent of the available energy surplus. It is well possible that other similar wave packages were excited in other regions not directly observed in-situ by the spacecraft so this is likely a conservative estimate of the cross-scale energy transport. Assuming the fast magnetosonic mode was completely damped, its wave energy was sufficient to cause $\sim 2 \mathrm{keV}$ 
increase in the ion energy when using the observed integrated kinetic energy flux magnitude during the wave observation. This cross-scale coupling mechanism could be applicable to broad range of systems that experience velocity shear and where KHI can form. For example, similar cross-scale mechanisms may contribute to the heating of the solar corona, where KH waves have been observed (Foullon et al. 2011; Ofman and Thompson 2011; Nykyri and Foullon 2013).

In addition to the mechanism described above, the ultra-low frequency waves (below $0.5 \mathrm{~Hz}$ ) are associated with mode conversion (Lee et al. 1994; Belmont et al. 1995; De Keyser et al. 1999). Johnson et al. (2001) showed that perpendicular wave power enhancement can be explained by mode conversion of compressional magnetohydrodynamic (MHD) waves, found in the magnetosheath, into Kinetic Alfvén Waves (KAWs) at the magnetopause. These KAWs have been expected to be important for plasma heating and mixing in the LLBL (Johnson and Cheng 1997, 2001). Chaston et al. (2007) have shown observations consistent with mode conversion from surface to KAWs and transport of both electromagnetic energy and plasma at the Alfvén resonance, at the dusk flank magnetopause, under northward IMF (see Fig. 2). However, please note that the mode conversion from surface waves to KAWs, as reported by Chaston et al. (2007), was originally proposed by Hasegawa (JGR, 1976). This mechanism would work even without ultra-low frequency waves in the magnetosheath or in the solar wind.

In addition to the magnetosonic mode, Moore et al. (2016) found waves close to Alfvén resonance that were consistent with the kinetic Alfvén waves (see Fig. 3). A recent statistical study by Moore et al. (2017) demonstrates that during KH events there exist more oblique ion scale waves with larger Poynting flux, when compared to boundary crossings without Kelvin-Helmholtz activity. Statistics of the plasma parameters during wave observations indicated that during KHI the ion scale wave intervals were associated with more plasma heating when compared to ion scale wave intervals without $\mathrm{KH}$ activity. These results strongly suggest that KHI is linked with enhanced growth of the KAWs and magnetosonic waves which in turn can heat ions effectively.

Finally, the Kelvin-Helmholtz Instability can generate thin current sheets where magnetic reconnection can occur (Nykyri and Otto 2001, 2004; Nykyri et al. 2006; Hasegawa et al. 2009; Eriksson et al. 2016; Li et al. 2016). Nykyri et al. (2006) observed ion beams during intervals where reconnection criteria were satisfied. While magnetic reconnection can also directly heat plasma particles, in particular when magnetosheath beta is low (Ma et al. 2014a, 2014b), it is possible that these ion beams associated with magnetic reconnection could also generate electromagnetic waves which in turn could heat the ions.

Above, we have described possible cross-scale energy transport mechanism associated with the KHI. These can be summarized as follows:

Mechanism 1: Velocity shear $->$ macroscopic KHI $->$ boundary layer with strong gradients and thin current sheets $->$ shell-like ion distributions $->$ fast magnetosonic waves $->$ ion heating.

Mechanism 2: Velocity shear -> macroscopic KHI -> mode conversion -> kinetic Alfvén waves $->$ ion heating.

Mechanism 3: Velocity shear $->$ macroscopic KHI $->$ boundary layer with strong gradients and thin current sheets $->$ magnetic reconnection $->$ ion heating, ion beams $->$ electromagnetic ion-scale waves $->$ more ion heating. 


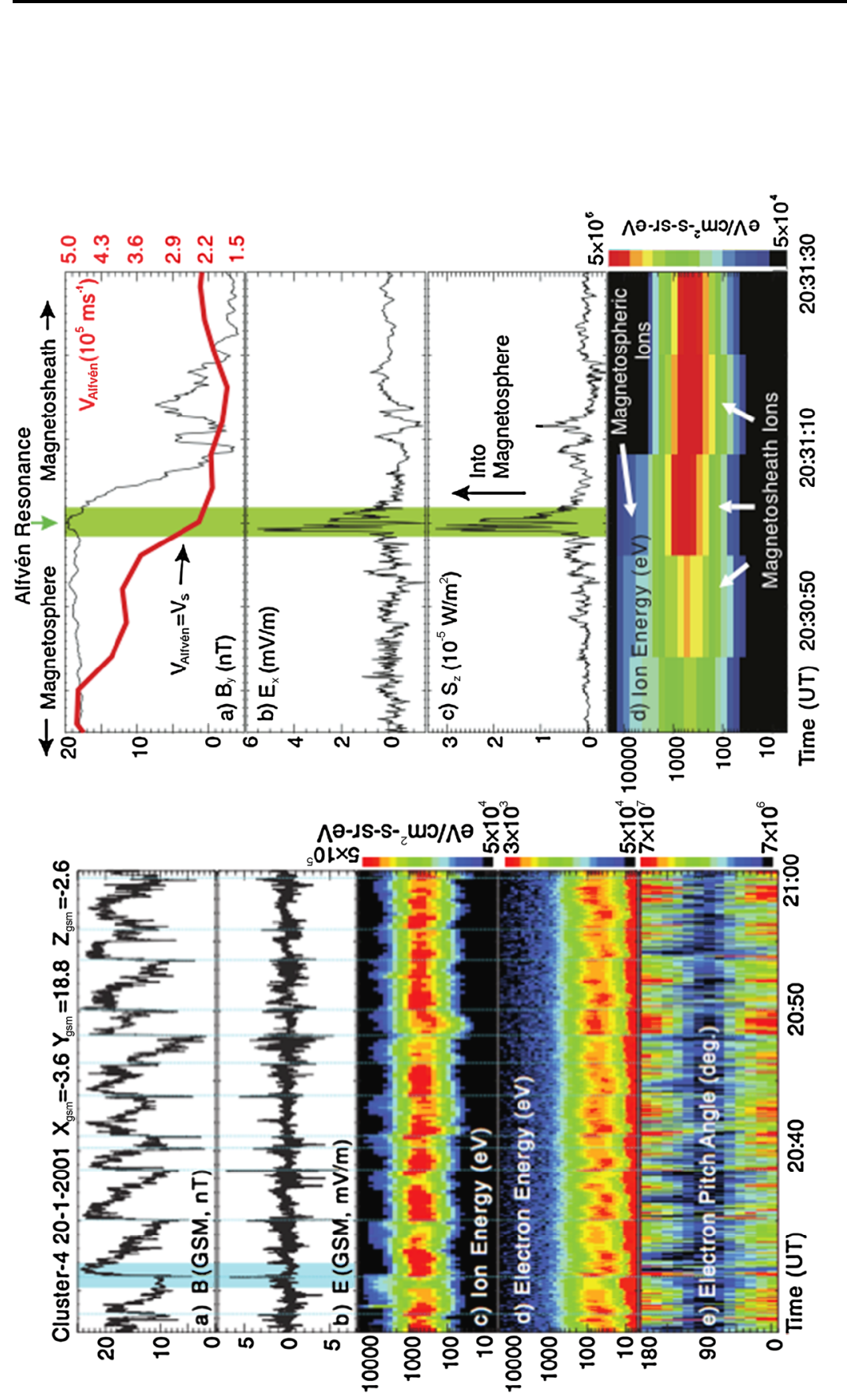

छ ฮ

㱐

क्ष

छิ

वี

急

远

范

密突

总告

ङ

궁

$\otimes 2$

记

하웡

政

$\pm$

$\Xi \approx$

o s

है

긍

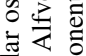

Б्.

몸

品

글

옹흘

就

जै

ธี

呵

U.

$\Xi$ 至区

몽

ㄴㅇㅇㅇㅇ

ฮิ

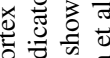

․․

玤总

๘

पै

ปิ)

可

D

응

屯․ำ

号苞

휴월

豞

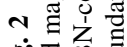

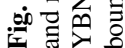




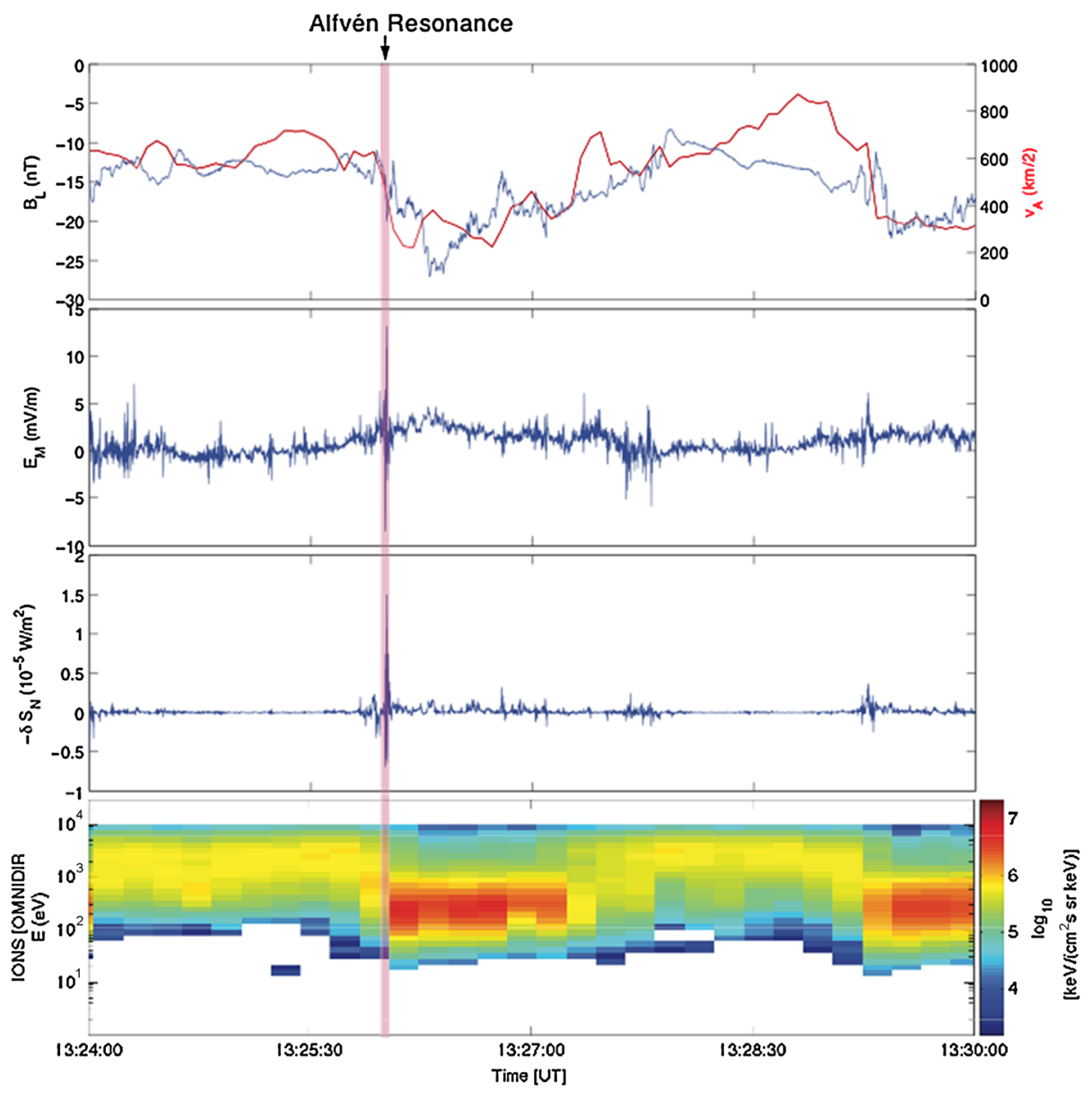

Fig. 3 A 6-minute time interval of the Moore et al. (2016) event in the same format as RHS of Fig. 2, showing enhanced Poynting flux into the magnetosphere at Alfvén resonance from C3

\section{Upcoming observation campaigns with MMS, cluster and THEMIS}

\subsection{Constellation of Spacecraft Constellations}

By June 2019, the orbits of the Cluster, MMS and THEMIS will have largely evolved (see Fig. 4). Pending the extension of Cluster operations in 2019, this mission will then make multi-scale observations along the magnetopause, while MMS will observe at electron scales more than $12 \mathrm{R}_{E}$ downstream. Hence, both missions may capture some of the changes occurring in $\mathrm{K}-\mathrm{H}$ waves over distances of more than $12 \mathrm{R}_{E}$ along the magnetopause. This kind of observations will enable, for the first time, to capture the evolution of the $\mathrm{KH}$ waves at separated points along the magnetopause. Cluster could observe the waves beginning to roll-up into vortices and the vortices as they develop and collapse, while MMS could study magnetic reconnection taking place in their twisted magnetic fields. Data from the ARTEMIS spacecraft at lunar orbit may also potentially be useful to capture the evolution of the KH waves (Sibeck et al. 2011). Meanwhile, the THEMIS constellation of three spacecraft will 
Fig. 4 Cluster, MMS, THEMIS and Swarm orbits in June 2019

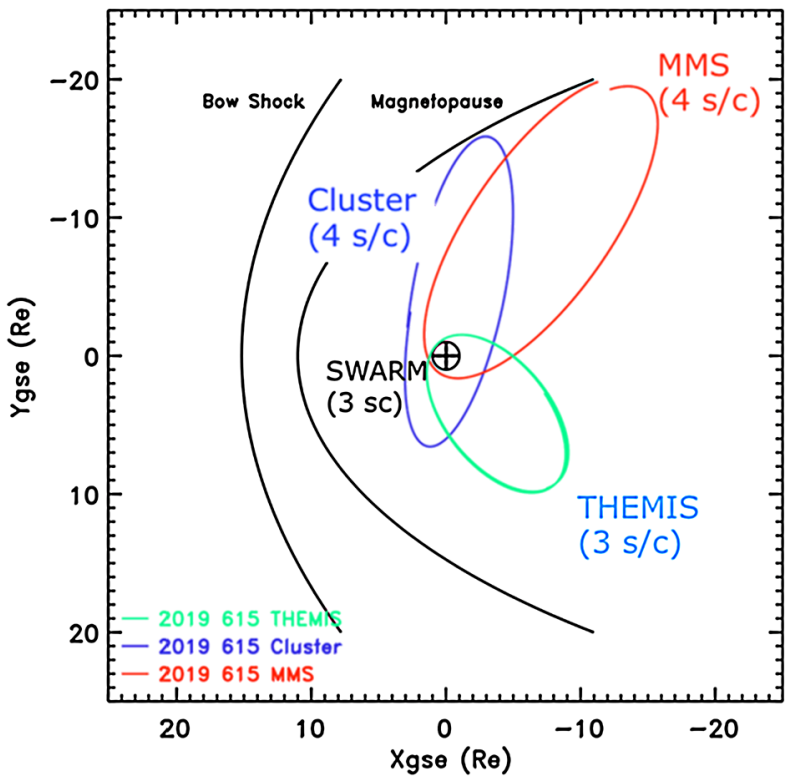

be located within the near Earth magnetotail. Consequently, it will be able to monitor the state of the magnetotail plasma sheet, and detect if CDPS events occur. Such events are characterized in particular by the electron density to be a factor 10 higher than usual.

In other words, these new and unique observations will enable to tackle the following open questions

- What is the evolution of a Kelvin-Helmholtz vortex from birth to collapse?

- How is magnetic reconnection initiated within $\mathrm{K}-\mathrm{H}$ rolled-up vortices?

- Do they enable solar wind plasma to enter into magnetospheres?

- What is their role in the formation and existence of the cold dense plasma sheet?

Furthermore, the ESA Swarm mission, with three spacecraft orbiting at around 400$500 \mathrm{~km}$ altitude, will seek the signature of $\mathrm{K}-\mathrm{H}$ waves in the high-latitude ionosphere, such as Field-Aligned Currents (FAC) associated with the relaxation of newly reconnected magnetic flux. K-H waves can also be observed on the ground in the form of ULF type pulsations in ground-based magnetometers (e.g. Pc3, Pc4, Pc5 or Ps6). The spacecraft observations will therefore be complemented with ground based observatories such as SuperMag but also THEMIS All Sky Imagers (ASI) network (e.g. detection of omega bands) and SuperDarn coherent radars network to scan the F-region (e.g. FAC maps).

\subsection{Dawn-Dusk Asymmetry}

The cold ions of magnetosheath origin are denser and hotter by 30-40 percent at the dawnside plasma sheet compared to those measured on the duskside (Hasegawa et al. 2003; Wing et al. 2005). A recent statistical study of magnetosheath temperatures using 7 years of THEMIS data indicates that ion magnetosheath temperatures downstream of quasi-parallel bow shock (dawn-flank for Parker-Spiral IMF) are only 10-15 percent higher than downstream of the quasi-perpendicular shock (Dimmock et al. 2015).

This magnetosheath temperature asymmetry (likely related to foreshock processes or insitu magnetosheath physics) is therefore inadequate to cause the observed level of the plasma 
sheet temperature asymmetry. Hence, additional physical mechanisms at the magnetopause must be at work to explain this asymmetric heating.

When studying the possible dawn-dusk asymmetries associated with the KHI and the physical mechanisms producing this, extreme care must be taken. This includes: location of the observed waves, possible orbital bias with respect to statistically most prevailing upstream shock geometry at dawn and dusk flanks, dipole tilt angle, presence of plasmaspheric plume (Walsh et al. 2015), magnetosheath seed fluctuations (Nykyri et al. 2017). For example, Taylor et al. (2012) observed more KH events at the dusk flank, but for many of these events, the IMF was in Ortho-parker Spiral orientation, making the magnetic field tension smaller on the dusk-sector.

Left panel of Fig. 5 presents the spatial distribution of the normalized occurrence rate of these KH waves observed between 2007-2013. It favours the dawn flank magnetopause under Parker-Spiral IMF orientation (Henry et al. 2017). MHD simulations demonstrate that for a variety of solar wind plasma conditions and under Parker-Spiral IMF orientation, the KHI growth at dawn-dusk terminator shows a slight preference for dawn flank due to the smaller magnetic field tension (see right panel of Fig. 5), when compared to the dusk flank (Nykyri 2013). Furthermore, a recent statistical study using 6 years of THEMIS data illustrates that the fluctuations in the ULF Pc4-Pc5 range, which characterizes the fluctuations generated by the KHI (but also other mechanisms such as FTE's and pressure pulses), is more enhanced in the dawn flank (Nykyri and Dimmock 2016). The spectral energy densities of the ion-scale electromagnetic waves are also more enhanced at the dawn-sector magnetopause (Yao et al. 2011). Recently, a statistical study using Cluster data has demonstrated that $\mathrm{KH}$ activity is associated with more energetic ion scale waves and enhanced ion heating when compared to boundary crossing without KHI (Moore et al. 2017). This suggests a positive correlation between $\mathrm{KH}$ waves and ion scale electromagnetic waves. The dawn-favoured asymmetry of the $\mathrm{KH}$ waves and ion scale waves may therefore explain the origin of the observed plasma sheet temperature and density asymmetry. In addition, the velocity and magnetic field seed fluctuations are more enhanced in the dawn magnetosheath (Dimmock et al. 2014, 2016) which may drive KHI more effectively in dawn-flank magnetopause (Nykyri et al. 2017). Data collected by MMS, THEMIS and the Van Allen Probes from May to August in 2018 may help to better understand this dawn-dusk asymmetry (see Fig. 6).

\subsection{KHI Turbulence}

Based on early MMS observations (September 2015), turbulence is present in the KHI, in the region between current sheets associated with the large-scale KHI (Stawarz et al. 2016). However, the presence of distinct periodic current sheets described in this paper may indicate that the turbulence is not fully developed. Once the apogee of the MMS spacecraft is raised, MMS will have the opportunity to cross the magnetopause farther down the tail with smaller spacecraft separation than in these early data ( $\sim 200 \mathrm{~km}$ inter-spacecraft separation). This will likely enable to probe turbulence in a much more developed state.

\subsection{Cross-Scale Energy Transport Within a KH Vortex}

The future studies with MMS spacecraft and future missions with cross-scale constellation spanning simultaneously the fluid, ion and electron scales can shed light on, which of the cross-scale coupling mechanisms described in Sect. 5 is the dominant one. Alternatively, there may be a dependence on upstream solar wind conditions and resulting magnetosheath 

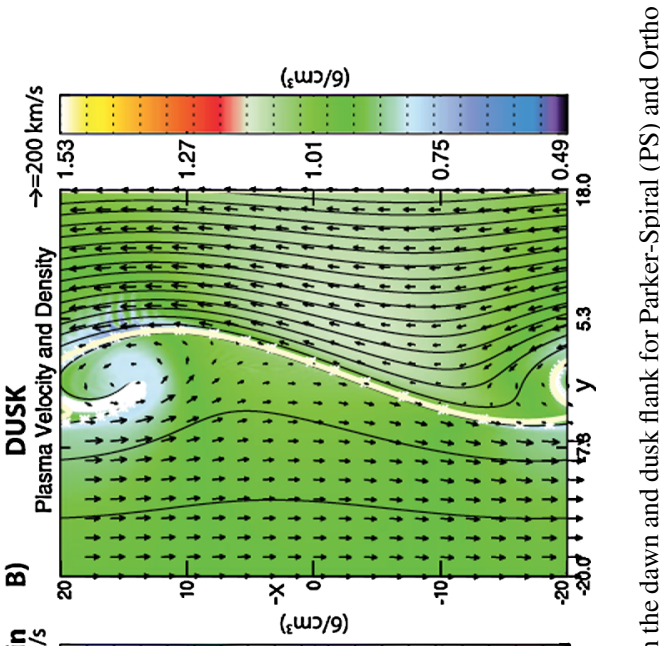

$\stackrel{2}{\frac{1}{2}}$

03

등

(2) 纪 雪 की Qิ

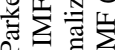
흥 잉 光昰

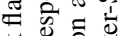

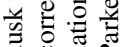
잉 ․ㅡㅇ 웅 灵声

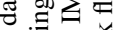
ฮ 氖

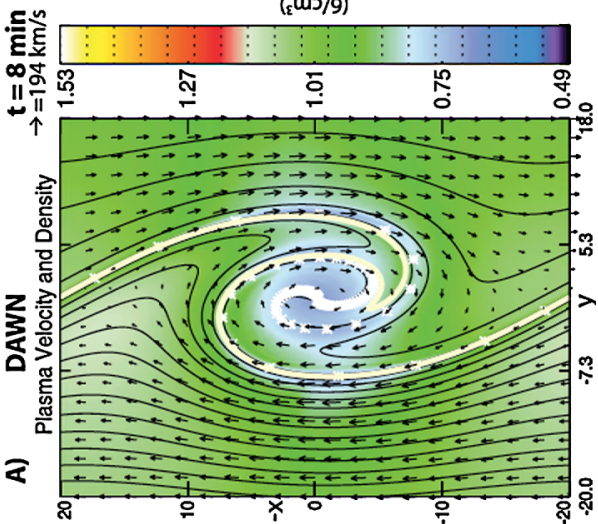
๙ิ 규율 ఫ 可 当虽 클 킁 क्षे ठ己

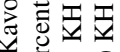
류류 Dै :

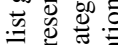
을 छี

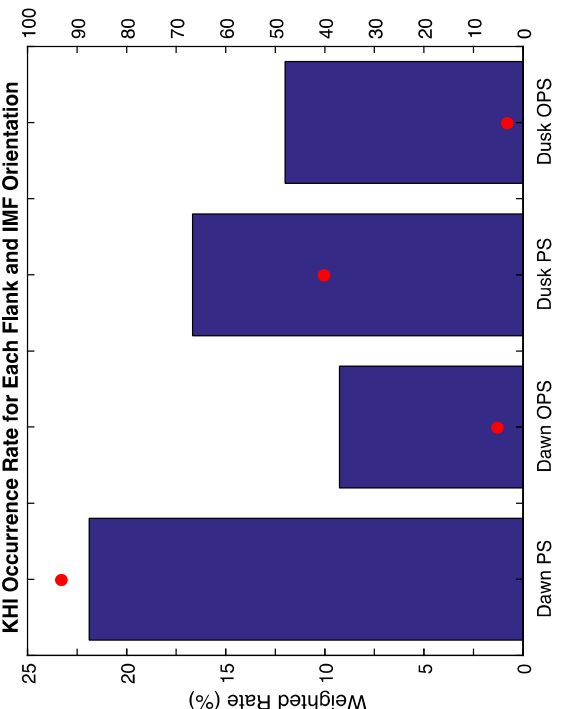

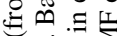
穴实 휴ㅇㅠㅛㅇㅠ ฮें D 式

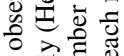

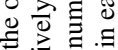

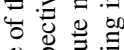
氜运㐘 छ సี

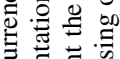
氖 矛

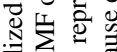
光光䒕

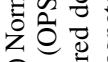
플 क की

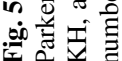


Fig. 6 Orbit configuration of MMS, THEMIS (THM) and the Van Allen Probes (VAP) in May-August 2018 timeframe

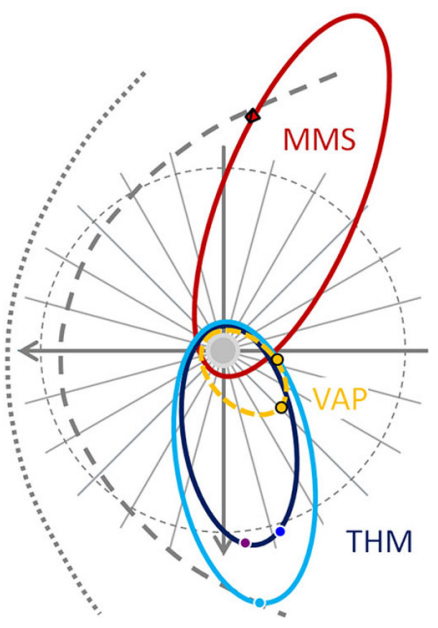

plasma parameters determining the efficiency of the particular cross-scale energy transport mechanism. A clue may be given by a relative constancy of the electron to ion temperature ratios (Lavraud et al. 2009). The dominant mechanism must be able to explain how the electrons "know" when ions have been heated. At higher $k$-values the ion-scale magnetosonic wave (which was shown to be able to energize the ions) is the whistler wave, so if there is sufficient wave energy left at higher $k$-value, this magnetosonic-whistler wave may be able to explain both the heating of ions and electrons during the KH events. The $10 \mathrm{~km}$ MMS spacecraft separations are ideal for studying the waves between ion and electron scales. With a bit of luck, an event may be found where the experimental dispersion relation, similar to the one found by Moore et al. (2016) may be extended to higher $k$-values and energy budget and transport can be quantitatively evaluated.

\section{Conclusion}

Since 2000, the Cluster and THEMIS missions have enabled a leap forward in our understanding of the Kelvin-Helmholtz instability thanks to unique multiple measurements at the magnetopause. For the first time, it was found that KHI can lead to the development of rolled-up vortices along the magnetopause. Statistical studies based on the data collected by these missions, but also Geotail and Double Star, have clearly shown that KHI is a common phenomenon. While more favoured under Northward IMF conditions, KHI was found to develop under any IMF conditions. Three main physical mechanisms have been identified to explain the cross-scale energy transport within a KHI vortex. Future coordinated observations in 2018-2020 by a unique set of multi-spacecraft missions (MMS, Cluster, THEMIS, Van Allen Probes, Swarm) may shed light on which one of these mechanisms is the dominant one. Other questions might hopefully be tackled by these upcoming measurements: how does this phenomenon develop from birth to collapse along the magnetopause? what is the role of turbulence if found in a fully developed state? Eventually these new observations will provide a more quantitative picture on a long-standing question: what is the contribution of the KHI vs. magnetic reconnection in terms of plasma entry depending on solar wind conditions. 
Open Access This article is distributed under the terms of the Creative Commons Attribution 4.0 International License (http://creativecommons.org/licenses/by/4.0/), which permits unrestricted use, distribution, and reproduction in any medium, provided you give appropriate credit to the original author(s) and the source, provide a link to the Creative Commons license, and indicate if changes were made.

\section{References}

E. Adamson, K. Nykyri, A. Otto, The Kelvin-Helmholtz instability under parker-spiral interplanetary magnetic field conditions at the magnetospheric flanks. Adv. Space Res. 58(2), 218-230 (2016)

V. Angelopoulos, The THEMIS mission. Space Sci. Rev. 141, 5 (2008). https://doi.org/10.1007/s11214008-9336-1

W.I. Axford, C.O. Hines, A unifying theory of high-latitude geophysical phenomena and geomagnetic storms. Can. J. Phys. 39, 1433 (1961)

W. Baumjohann, G. Paschmann, C.A. Cattell, Average plasma properties in the central plasma sheet. J. Geophys. Res. 94(A6), 6597-6606 (1989). https://doi.org/10.1029/JA094iA06p06597

G. Belmont, G. Chanteur, Advances in magnetopause Kelvin-Helmholtz instability studies. Phys. Scr. 40, 124 (1989)

G. Belmont, F. Reberac, L. Rezeau, Resonant amplification of magnetosheath MHD fluctuations at the magnetopause. Geophys. Res. Lett. 22, 295-298 (1995)

O. Berné, N. Marcelino, J. Cernicharo, Waves on the surface of the Orion molecular cloud. Nature 466, 947-949 (2010)

N. Bucciantini, E. Amato, L. Del Zanna, Relativistic MHD simulations of pulsar bow-shock nebulae. Astron. Astrophys. 434, 189-199 (2005). https://doi.org/10.1051/0004-6361:20042205

S. Chandrasekhar, Hydrodynamic and Hydromagnetic Stability (Oxford Univ. Press, Clarendon, 1961)

C.C. Chaston, M. Wilber, F.S. Mozer, M. Fujimoto, M.L. Goldstein, M. Acuna, H. Rème, A. Fazakerley, Mode conversion and anomalous transport in Kelvin-Helmholtz vortices and kinetic Alfvén waves at the Earth's magnetopause. Phys. Rev. Lett. 99(17), 175004 (2007)

Q. Chen, A. Otto, L.C. Lee, Tearing instability, Kelvin-Helmholtz instability, and magnetic reconnection. J. Geophys. Res. 102, 151-161 (1997)

S.G. Claudepierre, S.R. Elkington, M. Wiltberger, Solar wind driving of magnetospheric ULF waves: pulsations driven by velocity shear at the magnetopause. J. Geophys. Res. 113, A05218 (2008). https:// doi.org/10.1029/2007JA012890

M.M. Cowee, D. Winske, S.P. Gary, Two-dimensional hybrid simulations of superdiffusion at the magnetopause driven by Kelvin-Helmholtz instability. J. Geophys. Res. 114, A10209 (2009). https:// doi.org/10.1029/2009JA014222

M.M. Cowee, D. Winske, S.P. Gary, Hybrid simulations of plasma transport by Kelvin-Helmholtz instability at the magnetopause: density variations and magnetic shear. J. Geophys. Res. 115, A06214 (2010). https://doi.org/10.1029/2009JA015011

J. De Keyser, M. Roth, F. Reberac, L. Rezeau, G. Belmont, Resonant amplification of MHD waves in realistic subsolar magnetopause configurations. J. Geophys. Res. 104, 2399 (1999)

A.P. Dimmock, K. Nykyri, T.I. Pulkkinen, A statistical study of magnetic field fluctuations in the dayside magnetosheath and their dependence on upstream solar wind conditions. J. Geophys. Res. Space Phys. 119, 6231-6248 (2014). https://doi.org/10.1002/2014JA020009

A.P. Dimmock, K. Nykyri, H. Karimabadi, A. Osmane, T.I. Pulkkinen, A statistical study into the spatial distribution and dawn-dusk asymmetry of dayside magnetosheath ion temperatures as a function of upstream solar wind conditions. J. Geophys. Res. Space Phys. 120, 2767-2782 (2015). https://doi.org/10.1002/2014JA020734

A.P. Dimmock, K. Nykyri, A. Osmane, T.I. Pulkkinen, Statistical mapping of ULF Pc3 velocity fluctuations in the Earth's dayside magnetosheath as a function of solar wind conditions. Adv. Space Res. 58, 196-207 (2016). https://doi.org/10.1016/j.asr.2015.09.039

J.W. Dungey, Interplanetary magnetic field and auroral zones. Phys. Rev. Lett. 6, 47-48 (1961)

J.W. Dungey, The structure of the exosphere or adventures in velocity space, in Geophysics, the Earth's Environment, ed. by C. DeWitt, J. Hieblot, A. Lebeau (Gordon and Breach, Newark, 1963), p. 550

T.E. Eastman, E.W. Hones Jr., S.J. Bame, J.R. Asbridge, The magnetospheric boundary layer: site of plasma, momentum, and energy transfer from the magnetosheath into the magnetosphere. Geophys. Res. Lett. 3, 685 (1976)

S. Eriksson et al., Magnetospheric multiscale observations of magnetic reconnection associated with KelvinHelmholtz waves. Geophys. Res. Lett. 43, 5606-5615 (2016). https://doi.org/10.1002/2016GL068783 
C.P. Escoubet, M. Fehringer, M. Goldstein, The cluster mission. Ann. Geophys. 19(10/12), 1197-1200 (2001)

C.J. Farrugia, F.T. Gratton, L. Bender, H.K. Biernat, N.V. Erkaev, J.M. Quinn, R.B. Torbert, V. Dennisenko, Charts of joint Kelvin-Helmholtz and Rayleigh-Taylor instabilities at the dayside magnetopause for strongly northward interplanetary magnetic field. J. Geophys. Res. 103(A4), 6703-6727 (1998). https://doi.org/10.1029/97JA03248

C. Foullon, E. Verwichte, V.M. Nakariakov, K. Nykyri, C.J. Farrugia, Magnetic Kelvin-Helmholtz instability at the Sun. Astrophys. J. Lett. 729(1), L8 (2011)

M. Fujimoto, T. Terasawa, T. Mukai, Space Sci. Rev. 80, 325 (1997). https://doi.org/10.1023/ A:1004934306623

K. Grygorov, Z. Nemecek, J. Šafránková, L. Prech, G. Pi, J.-H. Shue, Kelvin-Helmholtz wave at the subsolar magnetopause boundary layer under radial IMF. J. Geophys. Res. Space Phys. 121, 9863-9879 (2016). https://doi.org/10.1002/2016JA023068

A. Hasegawa, Particle acceleration by MHD surface wave and formation of aurora. J. Geophys. Res. 81(28), 5083-5090 (1976). https://doi.org/10.1029/JA081i028p05083

H. Hasegawa, Structure and dynamics of the magnetopause and its boundary layers. Monogr. Environ. Earth Planets 1, 71-119 (2012). https://doi.org/10.5047/meep.2012.00102.0071

H. Hasegawa, M. Fujimoto, K. Maezawa, Y. Saito, T. Mukai, Geotail observations of the dayside outer boundary region: interplanetary magnetic field control and dawn-dusk asymmetry. J. Geophys. Res. 108(A4), 1163 (2003). https://doi.org/10.1029/2002JA009667

H. Hasegawa, M. Fujimoto, T.D. Phan, H. Rème, A. Balogh, M.W. Dunlop, C. Hashimoto, R. TanDokoro, Transport of solar wind into Earth's magnetosphere through rolled-up Kelvin-Helmholtz vortices. Nature 430, 755-758 (2004)

H. Hasegawa, M. Fujimoto, K. Takagi, Y. Saito, T. Mukai, H. Rème, Single-spacecraft detection of rolled-up Kelvin-Helmholtz vortices at the flank magnetopause. J. Geophys. Res. 111, A09203 (2006)

H. Hasegawa et al., Kelvin-Helmholtz waves at the Earth's magnetopause: multiscale development and associated reconnection. J. Geophys. Res. 114, A12207 (2009). https://doi.org/10.1029/2009JA014042

H.L.F. Helmholtz, On the discontinuous movements of fluids. Sitz.ber. Preuss. Akad. Wiss. Berl. Philos.-Hist. K1. 23, 215-228 (1868)

Z.W. Henry, K. Nykyri, T.W. Moore, A.P. Dimmock, X. Ma, On the dawn-dusk asymmetry of the KelvinHelmholtz instability between 2007-2013. J. Geophys. Res. 122, 11888 (2017). https://doi.org/10.1002/ 2017JA024548

R. Houze, Cloud Dynamics, 2nd edn. (Elsevier, Amsterdam, 2014). ISBN:9780123742667

K.-J. Hwang, M.M. Kuznetsova, F. Sahraoui, M.L. Goldstein, E. Lee, G.K. Parks, Kelvin-Helmholtz waves under southward interplanetary magnetic field. J. Geophys. Res. 116, A08210 (2011). https://doi.org/ 10.1029/2011JA016596

K.-J. Hwang, M.L. Goldstein, M.M. Kuznetsova, Y. Wang, A.F. Viñas, D.G. Sibeck, The first in situ observation of Kelvin-Helmholtz waves at high-latitude magnetopause during strongly dawnward interplanetary magnetic field conditions. J. Geophys. Res. 117, A08233 (2012). https://doi.org/10.1029/ 2011JA017256

J.R. Johnson, C.Z. Cheng, Kinetic Alfvén waves and plasma transport at the magnetopause. Geophys. Res. Lett. 24, 1423-1426 (1997). https://doi.org/10.1029/97GL01333

J.R. Johnson, C.Z. Cheng, Stochastic ion heating at the magnetopause due to kinetic Alfvén waves. Geophys. Res. Lett. 28, 4421-4424 (2001). https://doi.org/10.1029/2001GL013509

J.R. Johnson, C.Z. Cheng, P. Song, Signatures of mode conversion and kinetic Alfvén waves at the magnetopause. Geophys. Res. Lett. 28(2), 227-230 (2001). https://doi.org/10.1029/2000GL012048

J.R. Johnson, S. Wing, P.A. Delamere, Space Sci. Rev. 184, 1 (2014). https://doi.org/10.1007/s11214-0140085-Z

S. Kavosi, J. Raeder, Ubiquity of Kelvin-Helmholtz waves at Earth's magnetopause. Nat. Commun. 6, 7019 (2015). https://doi.org/10.1038/ncomms8019

H. Kawano, S. Kokubun, Y. Yamamoto, K. Tsuruda, H. Hayakawa, M. Nakamura, T. Okada, A. Matsuoka, A. Nishida, Magnetopause characteristics during a four-hour interval of multiple crossings observed with GEOTAIL. Geophys. Res. Lett. 21, 2895 (1994)

L. Kelvin (W.T. Thomson), Hydrokinetic solutions and observations. Philos. Mag. 42, 362-377 (1871)

B. Lavraud et al., Tracing solar wind plasma entry into the magnetosphere using ion-to-electron temperature ratio. Geophys. Res. Lett. 36, L18109 (2009). https://doi.org/10.1029/2009GL039442

L.C. Lee, J.R. Johnson, Z.W. Ma, Kinetic Alfvén waves as a source of plasma transport at the dayside magnetopause. J. Geophys. Res. 99, 17405 (1994)

W. Li et al., Kinetic evidence of magnetic reconnection due to Kelvin-Helmholtz waves. Geophys. Res. Lett. 43, 5635-5643 (2016). https://doi.org/10.1002/2016GL069192 
D. Lin, C. Wang, W. Li, B. Tang, X. Guo, Z. Peng, Properties of Kelvin-Helmholtz waves at the magnetopause under northward interplanetary magnetic field: statistical study. J. Geophys. Res. Space Phys. 119, 7485 (2014). https://doi.org/10.1002/2014JA020379

A.P. Lobanov, J.A. Zensus, A cosmic double helix in the archetypical quasar 3C273. Science 294, 128-131 (2001). https://doi.org/10.1126/science.1063239

X. Ma, A. Otto, P.A. Delamere, Interaction of magnetic reconnection and Kelvin-Helmholtz modes for large magnetic shear: 1. Kelvin-Helmholtz trigger. J. Geophys. Res. Space Phys. 119, 781-797 (2014a)

X. Ma, A. Otto, P.A. Delamere, Interaction of magnetic reconnection and Kelvin-Helmholtz modes for large magnetic shear: 2. Reconnection trigger. J. Geophys. Res. Space Phys. 119, 808-820 (2014b)

X. Ma, A. Otto, P. Delamere, H. Zhang, Interaction between reconnection and Kelvin-Helmholtz at the highlatitude magnetopause. Adv. Space Res. 58(2), 231-239 (2016)

Y. Matsumoto, M. Hoshino, Onset of turbulence induced by a Kelvin-Helmholtz vortex. Geophys. Res. Lett. 31, L02807 (2004). https://doi.org/10.1029/2003GL018195

V.V. Mishin, V.M. Tomozov, Sol. Phys. 291, 3165 (2016). https://doi.org/10.1007/s11207-016-0891-4

D.G. Mitchell et al., An extended study of the low-latitude boundary layer on the dawn and dusk flanks of the magnetosphere. J. Geophys. Res. 92(A7), 7394-7404 (1987)

A. Miura, P.L. Pritchett, Nonlocal stability analysis of the MHD Kelvin-Helmholtz instability in a compressible plasma. J. Geophys. Res. 87(A9), 7431-7444 (1982). https://doi.org/10.1029/JA087iA09p07431

T. Moore, Identifying signatures of plasma waves and reconnection associated with Kelvin-Helmholtz instabilities, ProQuest Dissertations and Theses, Thesis (M.S.E.P.), Embry-Riddle Aeronautical University, Daytona Beach, Fla (2012)

T.W. Moore, K. Nykyri, A.P. Dimmock, Cross-scale energy transport in space plasmas. Nat. Phys. 12, 11641169 (2016). https://doi.org/10.1038/nphys3869

T.W. Moore, K. Nykyri, A.P. Dimmock, Ion-scale wave properties and enhanced ion heating across the low-latitude boundary layer during Kelvin-Helmholtz instability. J. Geophys. Res. 122, 11128 (2017). https://doi.org/10.1002/2017JA024591

F.S. Mozer, H. Hayakawa, S. Kokubun, M. Nakamura, T. Okada, T. Yamamoto, K. Tsuruda, The morningside low-latitude boundary layer as determined from electric and magnetic field measurements on geotail. Geophys. Res. Lett. 21, 2983 (1994)

T.K.M. Nakamura, D. Hayashi, M. Fujimoto, I. Shinohara, Decay of MHD-scale Kelvin-Helmholtz vortices mediated by parasitic electron dynamics. Phys. Rev. Lett. 92, 145001 (2004)

M.N. Nishino, H. Hasegawa, M. Fujimoto, Y. Saito, T. Mukai, I. Dandouras, H. Rème, A. Retinò, R. Nakamura, E. Lucek, S.J. Schwartz, A case study of Kelvin-Helmholtz vortices on both flanks of the Earth's magnetotail. Planet. Space Sci. 59, 502-509 (2011)

K. Nykyri, Impact of MHD shock physics on magnetosheath asymmetry and Kelvin-Helmholtz instability. J. Geophys. Res. Space Phys. 118, 5068-5081 (2013). https://doi.org/10.1002/jgra.50499

K. Nykyri, A.P. Dimmock, Statistical study of the ULF Pc4-Pc5 range fluctuations in the vicinity of Earth's magnetopause and correlation with the low latitude boundary layer thickness. Adv. Space Res. 58, 257267 (2016)

K. Nykyri, C. Foullon, First magnetic seismology of the CME reconnection outflow layer in the low corona with 2.5-D MHD simulations of the Kelvin-Helmholtz instability. Geophys. Res. Lett. 40, 4154-4159 (2013). https://doi.org/10.1002/grl.50807

K. Nykyri, A. Otto, Plasma transport at the magnetospheric boundary due to reconnection in KelvinHelmholtz vortices. Geophys. Res. Lett. 28, 3565-3568 (2001)

K. Nykyri, A. Otto, Influence of the Hall term on KH instability and reconnection inside KH vortices. Ann. Geophys. 22, 935-949 (2004). https://doi.org/10.5194/angeo-22-935-2004

K. Nykyri, A. Otto, B. Lavraud, C. Mouikis, L.M. Kistler, A. Balogh, H. Rème, Cluster observations of reconnection due to the Kelvin-Helmholtz instability at the dawnside magnetospheric flank. Ann. Geophys. 24, 2619-2643 (2006). https://doi.org/10.5194/angeo-24-2619-2006

K. Nykyri, X. Ma, A. Dimmock, C. Foullon, A. Otto, A. Osmane, Influence of velocity fluctuations on the Kelvin-Helmholtz instability and its associated mass transport. J. Geophys. Res. Space Phys. 122, 9489 (2017). https://doi.org/10.1002/2017JA024374

L. Ofman, B.J. Thompson, SDO/AIA observation of Kelvin-Helmholtz instability in the solar corona. Astrophys. J. 734, L11 (2011)

F. Plaschke, M.G.G.T. Taylor, R. Nakamura, Alternative interpretation of results from Kelvin-Helmholtz vortex identification criteria. Geophys. Res. Lett. 41, 244-250 (2014). https://doi.org/10.1002/ 2013GL058948

K. Rönmark, WHAMP-waves in homogeneous, anisotropic, multicomponent plasmas. Tech. Rep. 179, Kiruna Geophysical Institute (1982)

D.G. Sibeck, V. Angelopoulos, D.A. Brain et al., ARTEMIS science objectives. Space Sci. Rev. 165, 59 (2011). https://doi.org/10.1007/s11214-011-9777-9 
R. Smets, D. Delcourt, G. Chanteur, T.E. Moore, On the incidence of the Kelvin-Helmholtz instability for mass exchange process at the Earth's magnetopause. Ann. Geophys. 20, 757-769 (2002)

W.D. Smyth, J.N. Moum, Ocean mixing by Kelvin-Helmholtz instability. Oceanography 25(2), 140-149 (2012). https://doi.org/10.5670/oceanog.2012.49

P. Song, C.T. Russell, Model of the formation of the low-latitude boundary layer for strongly northward interplanetary magnetic field. J. Geophys. Res. 97(A2), 1411-1420 (1992). https://doi.org/10.1029/ 91JA02377

J.E. Stawarz, S. Eriksson, F.D. Wilder, R.E. Ergun, S.J. Schwartz, A. Pouquet, J.L. Burch, B.L. Giles, Y. Khotyaintsev, O. Le Contel, P.-A. Lindqvist et al., Observations of turbulence in a Kelvin-Helmholtz event on 8 September 2015 by the Magnetospheric Multiscale mission. J. Geophys. Res. Space Phys. 121, 11021 (2016). https://doi.org/10.1002/2016JA023458

K. Takagi, C. Hashimoto, H. Hasegawa, M. Fujimoto, R. TanDokoro, Kelvin-Helmholtz instability in a magnetotail flank-like geometry: three-dimensional MHD simulations. J. Geophys. Res. 111, A08202 (2006). https://doi.org/10.1029/2006JA011631

M.G.G.T. Taylor, B. Lavraud, C.P. Escoubet, S.E. Milan, K. Nykyri, M.W. Dunlop, J.A. Davies, R.H.W. Friedel, H. Frey, Y.V. Bogdanova, A. Åsnes, H. Laakso, P. Travnicek, A. Masson, H. Opgenoorth, C. Vallat, A.N. Fazakerley, A.D. Lahiff, C.J. Owen, F. Pitout, Z. Pu, C. Shen, Q.G. Zong, H. Rème, J. Scudder, T.L. Zhang, The plasma sheet and boundary layers under northward IMF: a multi-point and multi-instrument perspective. Adv. Space Res. 41, 1619-1629 (2008). https://doi.org/10.1016/j.asr. 2007.10.013

M.G.G.T. Taylor, H. Hasegawa, B. Lavraud, T. Phan, C.P. Escoubet, M.W. Dunlop, Y.V. Bogdanova, A.L. Borg, M. Volwerk, J. Berchem, O.D. Constantinescu, J.P. Eastwood, A. Masson, H. Laakso et al., Spatial distribution of rolled up Kelvin-Helmholtz vortices at Earth's dayside and flank magnetopause. Ann. Geophys. 30, 1025-1035 (2012). https://doi.org/10.5194/angeo-30-1025-2012

B.M. Walsh, E.G. Thomas, K.-J. Hwang, J.B.H. Baker, J.M. Ruohoniemi, andJ.W. Bonnell, Dense plasma and Kelvin-Helmholtz waves at Earth's dayside magnetopause. J. Geophys. Res. Space Phys. 120, 55605573 (2015). https://doi.org/10.1002/2015JA021014

S. Wing, J.R. Johnson, P.T. Newell, C.-I. Meng, Dawn-dusk asymmetries, ion spectra, and sources in the northward interplanetary magnetic field plasma sheet. J. Geophys. Res. 110, A08205 (2005). https:// doi.org/10.1029/2005JA011086

G.Q. Yan, F.S. Mozer, C. Shen, T. Chen, G.K. Parks, C.L. Cai, J.P. McFadden, Kelvin-Helmholtz vortices observed by THEMIS at the duskside of the magnetopause under southward interplanetary magnetic field. Geophys. Res. Lett. 41, 4427-4434 (2014)

Y. Yao, C.C. Chaston, K.-H. Glassmeier, V. Angelopoulos, Electromagnetic waves on ion gyro-radii scales across the magnetopause. Geophys. Res. Lett. 38, L09102 (2011). https://doi.org/10.1029/ 2011GL047328 